\title{
《西北地质》编辑委员会 \\ Editorial Committee of Northwestern Geology
}

\section{主 任 Director of Editorial Committee 李文波 LI Wen-yuan \\ 副主任 Deputy Director of Editorial Committee \\ 丰成友 FENG Chengyou 章雨旭 ZHANG Yuxu}

顾 问 Counselor of Editorial Committee(按姓氏汉语拼音字母排序)

安芷生 AN Zhisheng 陈毓川 CHEN YUchuan

侯增谦 HOU Zengqian 金之钧 JIN Zhijun

林学钥 LIN Xuyyu毛景文 MAO Jingwen

汤中立 TANG Zhongli 王双明 WANG Shuangming

徐义刚 XU Yigang 杨经绥 YANG Jingsui

袁道先 YUAN Daoxian 张国伟 ZHANG Guowei

翟明国 ZHAI Mingguo 郑永飞 ZHENG Yongfeng

委 员 Members of Editorial Committee(按姓氏汉语拼音字母排序)

\begin{tabular}{|c|c|c|c|}
\hline 陈华勇 & CHEN Huayong & 陈隽璐 & CHEN Junlu \\
\hline 邓 军 & DENG Jun & 董福辰 & DONG Fuchen \\
\hline 丰成友 & FENG Chengyou & \multicolumn{2}{|c|}{ Franco Pirajno } \\
\hline 高永宝 & GAO Yongbao & 计文化 & JI Wenhua \\
\hline 李凤棠 & LI Fengtang & 李三忠 & LI Sanzhong \\
\hline 李献华 & LI Xianhua & 李玉宏 & LI Yuhong \\
\hline 吕新彪 & LV Xinbiao & 孟兴民 & MENG Xingmin \\
\hline 庞雄奇 & PANG Xiongqi & 裴先治 & PEI Xianzhi \\
\hline 钱壮志 & QIAN Zhuangzhi & 秦克章 & QIN Kezhang \\
\hline \multicolumn{2}{|c|}{ Tahseenullah Khan } & 唐辉明 & TANG Huiming \\
\hline \multicolumn{2}{|c|}{ Tom Dijkstra } & 万 力 & WAN Li \\
\hline 王家鼎 & WANG Jiading & 王 涛 & WANG Táo \\
\hline 王旭升 & WANG Xusheng & 王训练 & WANG Xunlian \\
\hline 王永和 & WANG Yonghe & 肖文交 & XIAO Wenjiao \\
\hline 强 & XU Qiang & 徐学义 & XU Xueyi \\
\hline 臻 & YAN Zhen & 杨志明 & YANG Zhiming \\
\hline 殷跃平 & YIN Yaoping & 张茂省 & ZHANG Maosheng \\
\hline 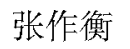 & ZHANG Zuoheng & 章雨旭 & ZHANG Yuxu \\
\hline 朱立新 & ZHU Lixin & 朱志新 & ZHU Zhixin \\
\hline
\end{tabular}

主 编 Editor in Chief 徐学义 XU Xueyi

副主编 Deputy Editor in Chief

丰成友 FENG Chengyou

计文化 JI Wenhua
张茂省 ZHANG Maosheng

陈隽路 CHEN Junlu
陈祖显 CHEN Zuyu

李佩成 LI Peicheng

舒德干 SHU Degan

夏林圻 XIA Linqi

杨树锋 YANG Shufeng

张宏福 ZHANG Hongfu

陈宣华 CHEN Xuanhua

董云鹏 DONG Yunpeng

高晓峰 GAO Xiaofeng

赖绍聪 LAI Shaocong

李文渊 LI Wenyuan

刘池阳 LIU Chiyang

倪 培 NI Pei

彭建兵 PENG Jianbing

孙丰月 SUN Fengyue

滕家欣 TENG Jiaxin

王登红 WANG Denghong

王文科 WANG Wenke

王焰新 WANG Yanxin

许 领 XU Ling

徐友宁 XU Youning

尹立河 YIN Lihe

张拴宏 ZHANG Shuanhong

周涛发 ZHOU Táfa

赖绍聪 LAI Shaocong

《西北地质》编辑部

Editorial Department of Northwestern Geology 